\title{
Isolation of the Dipolar Relaxation of Restricted Sets of Nuclear Spins
}

\author{
T. J. Norwood, ${ }^{*}$ J. Pereira, $\dagger$ And A. Chaudhry $*$ \\ * Department of Chemistry, Leicester University, University Road, Leicester LE1 7RH, United Kingdom; and $\dagger$ Instituto de Ciencias \\ Biomedicas de Abel Salazar, Universidade do Porto, Rua D. Manuel II, 4003 Porto Codex, Portugal \\ Received August 30, 1995; revised December 6, 1995
}

NMR relaxation parameters are potentially a valuable source of information on molecular dynamics. However, due to the complexity of the processes which can occur, studies are usually restricted to those cases in which relatively few processes can be considered to contribute to relaxation. These include ${ }^{15} \mathrm{~N}$ and ${ }^{13} \mathrm{C}$, but usually exclude the most abundant source of relaxation information, protons. General expressions are derived for all elements of the dipolar relaxation matrix that are valid for a system consisting of an arbitrary number of inequivalent weakly coupled spins. The similarities apparent between many of the diagonal elements are exploited to isolate the mutual relaxation of a restricted set of spins by taking linear combinations of relaxation rates. The experimental procedures necessary for making the measurements required to determine the mutual relaxation of a single pair of spins are discussed and are demonstrated for protons. ๑) 1996 Academic Press, Inc.

\section{INTRODUCTION}

NMR relaxation measurements are a valuable source of information on molecular dynamics $(1-6)$. Due to the complexity of the processes which can occur, such studies are usually restricted to circumstances in which the mutual relaxation of a small group of nuclear spins can be considered in isolation from all other interactions. This approach has seen wide use in studies of proteins which have been either uniformly ${ }^{15} \mathrm{~N}$ labeled or selectively ${ }^{13} \mathrm{C}$ labeled $(7-11)$. In each case, the heteroatom is considered to relax solely due to its own chemical-shift anisotropy and through dipolar interactions with its bonded protons. This method has the disadvantage that isotopic labeling is often required to achieve sufficient sensitivity, which can restrict the extent of its application.

We have recently showed that, in principle, dynamic information about a molecule can be obtained without the need to resort to labeling to obtain acceptable sensitivity by using proton-relaxation measurements, since, by taking a linear combination of the relaxation rates of a number of coherences or longitudinal modes, it is possible to isolate the mutual dipolar relaxation of a single pair of spins independently of any other interactions they might have $(12,13)$.
All interactions not between the chosen set of spins are made to undergo mutual cancellation. The mutual cancellation of some interactions is also implicit in work carried out by other groups to determine the value of the spectral-density function at a specific frequency (14). To determine unique values for a set of parameters associated with molecular motion, it is necessary to make an equivalent number of independent experimental measurements dependent on different linear combinations of the spectral-density function. The commonly used "model-free" approach has three parameters (15). We have previously demonstrated the isolation of the mutual relaxation of a single pair of spins as a function of two different linear combinations of the spectraldensity function $(12,13)$.

In this paper, we present generalized equations that can be used to write expressions for all elements of the dipolar relaxation matrix for an arbitrary number of weakly coupled inequivalent spins. Using these equations, we determine expressions for the relaxation rates of a number of coherence and longitudinal spin modes. These are used to determine linear combinations of relaxation rates that result in the isolation of the mutual relaxation of a restricted set of spins. Methods for making the required measurements for those combinations that isolate the mutual relaxation of a single pair of spins are discussed, and pulse sequences are presented for measuring the in-phase relaxation rates of zero-, single, and double-quantum coherence and the antiphase relaxation rate of single-quantum coherence. Experimental data are presented for 7-methoxycoumarin and 5-methylfurfural, and are used to investigate the motion of these molecules.

\section{GENERAL EXPRESSIONS FOR ELEMENTS OF THE DIPOLAR RELAXATION MATRIX}

The time dependence of the density operator can be written in matrix form as $(1-3)$

$$
d\{\boldsymbol{\sigma}(t)\} / d t=-\{i \mathscr{H}+\boldsymbol{\Gamma}\}\left\{\boldsymbol{\sigma}(t)-\boldsymbol{\sigma}_{0}\right\},
$$

where $\mathscr{H}$ is the time-independent nuclear spin superoperator, $\boldsymbol{\Gamma}$ is the relaxation superoperator, $\boldsymbol{\sigma}(t)$ is the density operator 